\title{
Asymmetric GARCH type models for asymmetric volatility characteristics analysis and wind power forecasting
}

\author{
Hao Chen $^{1^{*}}$, Jianzhong Zhang ${ }^{2}$, Yubo Tao ${ }^{1}$ and Fenglei $\operatorname{Tan}^{1}$
}

\begin{abstract}
Wind power forecasting is of great significance to the safety, reliability and stability of power grid. In this study, the GARCH type models are employed to explore the asymmetric features of wind power time series and improved forecasting precision. Benchmark Symmetric Curve (BSC) and Asymmetric Curve Index (ACI) are proposed as new asymmetric volatility analytical tool, and several generalized applications are presented. In the case study, the utility of the GARCH-type models in depicting time-varying volatility of wind power time series is demonstrated with the asymmetry effect, verified by the asymmetric parameter estimation. With benefit of the enhanced News Impact Curve (NIC) analysis, the responses in volatility to the magnitude and the sign of shocks are emphasized. The results are all confirmed to be consistent despite varied model specifications. The case study verifies that the models considering the asymmetric effect of volatility benefit the wind power forecasting performance.
\end{abstract}

Keywords: GARCH, Asymmetric GARCH model, News impact curve (NIC), Benchmark symmetric curve (BSC), Asymmetric curve index (ACl), Wind power forecasting

\section{Introduction}

On account of the lack of fossil resources and environment protection demand, wind power is becoming one of the most rapidly growing renewable energy sources, and regarded as an appealing alternative to conventional power generated from fossil fuel, which plays a very important role in national energy policies all around the world. Because of its advantages of clean, low carbon and renewable energy, wind power has provided strong support for energy saving, emission reduction and environmental protection in various countries $[1,2]$. According to the data released in 2017, it has been stated that the global installed wind power capacity has been 539 GW by the end of 2017 and a new record will be set in the future. Particularly, China, as the driver of global market growth for most of the last decade, increased its capacity by $19.7 \mathrm{GW}$ in 2017 . The wind power generation reached 305.7 TWh in 2017 [3]. However, the amount of power generation in wind farms usually show wide

\footnotetext{
* Correspondence: pingfengma@126.com

'State Grid Jiangsu Electric Power Co., Ltd Maintenance Branch Company, Nanjing 211102, Jiangsu, China

Full list of author information is available at the end of the article
}

fluctuations, therefore, it is difficult to forecast precisely and establish an accurate dispatch plan.

Various approaches have been applied in the forecasting of wind power [4-14]. A review of state-of-the-art approaches to wind speed forecasting has been published in [4]. Models used for wind speed forecasting can be usually categorized as physics-based models [5], statistical models, and spatial models [6], which includes ARIMA [7], Generalized Autoregressive Conditional Heteroskedasticity (GARCH) [8], Kalman filters [9] and more recent machine learning technologies such as neural networks $[10,11]$ and machine learning and deep learning methods $[12,13]$, are widely used in recent literature papers. Moreover, some ensemble approaches witness the improved the forecasting accuracy than the single methods [14].

The GARCH [8], usually has a better performance because traditional statistical methods like ARMA are built on linear assumptions and the nonlinear patterns hidden in the wind speed time series is hard to be captured. However, the research on the characteristics analysis of wind power time series is far from substantial. The GARCH model assumes that the response of volatility to a shock is a function of the shock strength only, without 
any correlation with the sign of the shock. In contrast, empirical evidence indicates that positive and negative shocks of equal strength may lead to different responses to the volatility in some practical time series. For example, due to the structure of a wind turbine, the influences of wind increasing and wind weakening is different. The standard GARCH model cannot depict this characteristic.

This paper focuses on the asymmetric characteristics in the volatility of wind power time series, which is very different from the related literature. The asymmetric GARCH models are employed to capture the asymmetric characteristics of volatility. Furthermore, the forecasting ability of GARCH type models is verified according to 3 statistical criteria.

Special attention is paid to the impact of news on the volatility of wind power time series with the News Impact Curve (NIC), which is presented in reference [15]. $\mathrm{NIC}$ is regarded as an efficient analytical tool to measure the extent of new information being incorporated into volatility estimates in many research domains. However, the classical NIC has some limitations in the analysis on the wind power time series with complicated volatility characteristics. Further modification and complementarities to refine the classical NIC are necessary. In this paper, two novel notions, including a Benchmark Symmetric Curve (BSC) and Asymmetric Curve Index (ACI), are proposed to provide an enhanced NIC analysis. Specifically, BSC is proposed to give a modest benchmark to the asymmetric NIC, and ACI is presented to quantitatively measure the asymmetry of NIC.

The paper is organized as follows. First, Section II presents a survey of asymmetric GARCH type models. Section III illustrates the extended notion of the NIC. Section IV demonstrates the results of a case study. With the refined NIC with a BSC and ACI, the responses in conditional variance of GARCH-type models are analyzed. Furthermore, the out-of-sample forecasting of GARCH type models is illustrated. At last, discussions are concluded in Section V.

\section{Asymmetric GARCH models}

\subsection{GARCH-M model}

GARCH model, an extended version of ARCH model [16], offers a general framework to model the heteroscedastic characteristic in time series [17].

The original GARCH $(p, q)$ model is formulated by (1) and (2) as below:

Conditional Mean:

$$
y_{t}=E\left(y_{t} \mid \Omega_{t-1}\right)+\varepsilon_{t}
$$

Conditional Variance:

$$
h_{t}=\alpha_{0}+\sum_{i=1}^{q} \alpha_{i} \varepsilon_{t-i}^{2}+\sum_{j=1}^{p} \beta_{j} h_{t-j}
$$

where, $\varepsilon_{t}=\sqrt{h_{t}} v_{t}$ is the residuals; $v_{t}$ is the innovation; $v_{t}$ $\sim$ I. I. D.,which follows $E\left(v_{t}\right)=0, E\left(v_{t}^{2}\right)=1$. $E\left(y_{t} \mid \Omega_{t-1}\right)$ is the expectation taking into account the information set, $\Omega_{t-1}$, dated $t-1$ and earlier. Non-negative integers, $p$ and $q$, are the order of the variance equation, and $\alpha_{0}>0, \alpha_{i} \geq 0$, $(i=1,2, \cdots q) ; \beta_{j} \geq 0,(j=1,2 \ldots p)$, respectively.

Moreover, considering the correlation of the volatility and the average value of wind power, which is called GARCH in mean effect [8], introducing a GARCH-M model to depict the wind power time series is a more appropriate choice.

A basic GARCH-M model is formulated by (3) and (2),

$$
y_{t}=\mu+g\left(h_{t}\right)-E\left(g\left(h_{t}\right)\right)+\varepsilon_{t}
$$

where, $h_{t}$ is defined as a GARCH process, given by (2), and $E\left(g\left(h_{t}\right)\right)$ is the expectation of $g\left(h_{t}\right)$.

The volatility compensation term $g\left(h_{t}\right)$ is a function with positive range which denotes the impact magnitude of volatility on the conditional mean equation. Practically, there are three popular hypothesises of $g\left(h_{t}\right)$ :

$$
\begin{aligned}
& g\left(h_{t}\right)=\delta h_{t} \\
& g\left(h_{t}\right)=\delta h_{t}^{1 / 2} \\
& g\left(h_{t}\right)=\delta \log h_{t}
\end{aligned}
$$

where, $\delta$ is the volatility compensation parameter that can describe the direct influence of the volatility term on the conditional mean of wind power time series.

\subsection{Asymmetric GARCH type models}

To accommodate these asymmetric characteristics of the volatility, various extensions of asymmetric GARCH type models are developed. These models include GJRGARCH [17], APARCH [18], and EGARCH [19], etc.

The above three models are employed in this study and introduced as follows. Note that each conditional mean equation of these models is defined as (3) with the volatility compensation term shown in (4), (5) or (6).

\section{1) GJR GARCH}

The conditional variance equation of GJR GARCH model is specified as

$$
h_{t}=\alpha_{0}+\sum_{j=1}^{p} \beta_{j} h_{t-j}+\sum_{i=1}^{q} \alpha_{i} \varepsilon_{t-i}^{2}+\gamma \varepsilon_{t-1}^{2} d_{t-1}
$$

where $\gamma$ denotes the asymmetric parameter; $d_{t-1}$ 
denotes a dummy variable when $\varepsilon_{t-1}<0, d_{t-1}=1$ and when $\varepsilon_{t-1} \geq 0, d_{t-1}=0$.

\section{2) $\mathrm{APARCH}$}

An asymmetric power ARCH (APARCH) model specifies $h_{t}$ as

$$
{ }_{h_{t}}^{\delta} / 2=\alpha_{0}+\sum_{j=1}^{p} \beta_{j} h_{t-j}^{\delta / 2}+\sum_{i=1}^{q} \alpha_{i}\left(\left|\varepsilon_{t-i}\right|-\gamma \varepsilon_{t-i}\right)^{\delta}
$$

where $\gamma$ is the asymmetric parameter and $\delta$ is the power term parameter.

It is probable to fit lots of GARCH type models within the APARCH model by assigning certain values to $\alpha, \beta$, $\gamma$ and $\delta$.

Especially, APARCH will be reduced to a $\mathrm{GARCH}$ model, if $\alpha, \beta$ is free, $d=2$ and $\gamma=0$.

\section{3) $\mathrm{EGARCH}$}

EGARCH is another popular model of asymmetric GARCH type. Conditional variance equation of an EGARCH model is defined as follows

$$
\ln h_{t}=\alpha_{0}+\sum_{j=1}^{p} \beta_{j} \ln h_{t-j}+\sum_{i=1}^{q}\left(\alpha_{i}\left|\frac{\varepsilon_{t-i}}{\sqrt{h_{t-i}}}\right|+\phi_{i} \frac{\varepsilon_{t-i}}{\sqrt{h_{t-i}}}\right)
$$

The asymmetric factor $\phi_{i}$ denotes the asymmetric effect to different shocks. The positive shocks bear stronger marked impact than the negative shocks if $\phi_{i}>0$, while the positive shocks contribute less volatility than negative shocks if $\phi_{i}<0$.

\subsection{Improved models considering non-Gaussian distribution}

In standard GARCH type models, the distribution of innovation is generally assumed to be the normal distribution. However, for many time series, this sort of assumption does not suit the excess of kurtosis. There exist lots of reports about the empirical conditional distribution with heavy tails [20,21].

In order to depict leptokurtosis more precisely, $t$-distribution and Generalized Error Distribution (GED) are selected as heavy tail alternative distributions.

The density of $t$-distribution is usually defined as:

$$
f(x, k)=\frac{\Gamma\left(\frac{k+1}{2}\right)}{(k \pi)^{\frac{1}{2}} \Gamma\left(\frac{k}{2}\right)}\left[1+\left(\frac{x^{2}}{k}\right)\right]^{\frac{-(k+1)}{2}}
$$

where $k>0, \Gamma(\cdot)$ denotes gamma function.
The density of a GED, normalized to mean $=0$ and variance $=1$, is expressed as

$$
f(x, v)=\frac{v \exp \left[-0.5 \cdot|x / \lambda|^{\nu}\right]}{\lambda \cdot 2^{(1+1 / v)} \Gamma\left(\frac{1}{v}\right)}
$$

where $\lambda$ is a constant which is defined as $\lambda=$ $\left[2^{-\frac{2}{v}} \Gamma(1 / v) / \Gamma(3 / v)\right]^{\frac{1}{2}}$, and $v$ denotes a shape parameter. Specially, for $v=2, x$ follows standard normal distribution. For $v<2$, tails of the distribution of $x$ are heavier than that of the normal distribution.

In this paper, the innovations with normal distribution $\left(v_{t} \stackrel{\text { i.i.d. }}{\sim} N(0,1)\right), t$-distribution $\left(v_{t}=\sqrt{(k-2) / k} x \stackrel{\text { i.i.d. }}{\sim} t(0,1\right.$ $; k))$ and $\operatorname{GED}\left(v_{t} \stackrel{i . i . d .}{\sim} \operatorname{GED}(0,1 ; v)\right)$ are integrated into asymmetric GARCH type models, respectively.

\subsection{Estimation}

In this work, Conditional Maximum Likelihood Estimate (CMLE) is introduced to specify asymmetric GARCH type model with the normal distribution, such as GJR, APARCH and EGARCH. A Marquardt algorithm, a popular refined version of a Gauss-Newton algorithm, is employed to optimize the iterative process.

Furthermore, by maximizing the log-likelihood function, the estimates of parameters in the heavy-tailed asymmetric GARCH type models can be also calculated, given the condition that $v_{t}$ follows $t$-distribution or GED for GJRGED, GJR-t, APARCH-t, APARCH-GED, EGARCH-t, and EGARCH-GED, respectively. A BHHH algorithm, instead of the Marquardt algorithm, is introduced to carry out the iterative process [21].

\section{The enhanced NIC analysis 3.1 The classical NIC}

Reference [14] proposed the news impact curve (NIC) as the recommended method to analyze asymmetry in how new information impacts the following period variance.

In classical NIC, by keeping the information dated $t-2$ and earlier as constant, the implicit relationship between $\varepsilon_{t-1}$ and $h_{t}$ can be highlighted. To simplify the problem, all lagged conditional variances are estimated at the numerical level of the unconditional variance.

In particular, for the standard GARCH model, the NIC is a symmetric curve with the quadratic function, which has a symmetry axis, $\varepsilon_{t-1}=0$. In the typical asymmetric GARCH model, the NIC is an asymmetric curve with the minimum at $\varepsilon_{t-1}=0$.

In this study, to simplify the discussion, the lowest point of the NIC is removed to the original point. For instance, the NIC of GJR $(1,1)$ model is shown as 


$$
\left\{\begin{array}{l}
h_{t}=\left(\alpha_{1}+\gamma\right) \varepsilon_{t-1}^{2}, \quad \text { if } \varepsilon_{t-1} \geq 0 \\
h_{t}=\alpha_{1} \varepsilon_{t-1}^{2}, \quad \text { if } \varepsilon_{t-1}<0
\end{array}\right.
$$

Note that the lagged conditional variance is estimated at its unconditional level.

\subsection{The benchmark symmetric curve (BSC)}

Since the NIC of standard GARCH is symmetric, it is often used as a benchmark to illustrate the asymmetry of other GARCH type models. Reference [14] uses the NIC of GARCH model as a benchmark; it is a good choice for GJR, but not for APARCH or another asymmetric GARCH models with complicated structure. That is to say, due to a variety in the left of variance eqs. (7-9), the shapes of NIC of GARCH type models are greatly different from this benchmark curve based on GARCH. In this case, the comparison with the NIC of GARCH might not be proper. A new form of the benchmark is derived as follows.

The conditional variance equation of a parametric asymmetric GARCH type model can be decomposed as

$$
g\left(h_{t}\right)=f(I)+\tilde{\eta}\left(\varepsilon_{t-1}, \cdots, \varepsilon_{t-i}, \cdots, \varepsilon_{t-q}\right)
$$

Considering the classical assumption of NIC, the news dated $t-2$ and earlier is held. Specification of (13) can be reduced to (14)

$$
g\left(h_{t}\right)=f(I)+\eta\left(\varepsilon_{t-1}\right)
$$

where $\eta\left(\varepsilon_{t-1}\right)$ is a function decided by $\varepsilon_{t-1}$, which has the asymmetric parameter to illustrate the asymmetry in volatility, while $f(I)$ has no relevance with $\varepsilon_{t}$.

Assigning the asymmetric parameter of the $\eta\left(\varepsilon_{t}\right)$ zero, the $\eta_{\text {even }}\left(\varepsilon_{t}\right)$ is obtained, which is obviously an even function of $\varepsilon_{t}$.

The Benchmark Symmetric Curve (BSC) of an asymmetric GARCH type model, noting as XGARCH, can be defined by

$$
H_{\mathrm{XGARCH}} \triangleq g\left(h_{t}\right)=f(I)+\eta_{\text {even }}\left(\varepsilon_{t-1}\right)
$$

where, $f(I)+\eta_{\text {even }}\left(\varepsilon_{t-1}\right)$ is a function decided by $\varepsilon_{t-1}$. And the $H-\varepsilon_{t-1}$ curve is the BSC of XGARCH model.

For example, the BSC of APARCH $(1,1)$ model can be derived as

$$
H_{A P A R C H}=h_{t}^{\delta / 2}=\alpha_{0}+\beta_{1} h_{t-1}^{\delta / 2}+\alpha_{1}\left|\varepsilon_{t-1}\right|^{\delta}
$$

\subsection{The asymmetric curve index $(\mathrm{ACl})$}

The asymmetric volatility brought by new information is explicitly recognized by the NIC. Though reference [21] proposed the method that can determine the level of asymmetry by the figure of the NIC, the quantitatively evaluating method is lacking. Asymmetric Curve Index
(ACI), which can give a quantitative judge to the extent of volatility asymmetry, is proposed in this work.

A parametric GARCH type model, either asymmetric or symmetric, can be decomposed as

$$
g\left(h_{t}\right)=f(I)+\eta\left(\varepsilon_{t}\right)
$$

where, $\eta\left(\varepsilon_{t}\right)$ is a function decided by $\varepsilon_{t}$, while $f(I)$ is irrelevant to $\varepsilon_{t}$.

Let $\varepsilon_{t}=E_{t}$, when $\varepsilon_{t}>0$, the $A C I$ is defined by

$$
A C I=\frac{\eta\left(E_{t}\right)-\eta\left(-E_{t}\right)}{\eta\left(E_{t}\right)+\eta\left(-E_{t}\right)}
$$

Obviously, if the GARCH type model has a symmetric structure, the $A C I$ is fixed at zero consequentially.

$$
A C I_{G A R C H}=\frac{\eta\left(E_{t}\right)-\eta\left(-E_{t}\right)}{\eta\left(E_{t}\right)+\eta\left(-E_{t}\right)}=\frac{0}{2 \eta\left(E_{t}\right)}=0
$$

Then, the novel ACIs are applied to some typical asymmetric GARCH type models.

For the GJR-GARCH $(1,1)$ model, the $A C I$ can be obtained as:

$$
\begin{aligned}
& \eta\left(E_{t}\right)=\alpha_{1} E_{t-1}^{2} \\
& \eta\left(-E_{t}\right)=\alpha_{1} E_{t-1}^{2}+\gamma E_{t-1}^{2}=\left(\alpha_{1}+\gamma\right) E_{t-1}^{2} \\
\{ & A C I_{G J R}=\frac{\eta\left(E_{t}\right)-\eta\left(-E_{t}\right)}{\eta\left(E_{t}\right)+\eta\left(-E_{t}\right)}=\frac{\alpha_{1}-\alpha_{1}-\gamma}{\alpha_{1}+\alpha_{1}+\gamma} \\
= & \frac{-\gamma}{2 \alpha_{1}+\gamma}
\end{aligned}
$$

Note that in the GJR GARCH model, the $A C I_{G J R}$ does not depend on $\mathrm{E}_{t-1}$. It just depends on the parameter of $\gamma$ and $\alpha_{1}$.

For the APARCH $(1,1)$ model, the $A C I$ can be expressed as

$$
\begin{gathered}
\eta\left(E_{t}\right)=\alpha_{1}\left((1-\gamma) E_{t-1}\right)^{\delta}=\alpha_{1}(1-\gamma)^{\delta}\left(E_{t-1}\right)^{\delta} \\
\eta\left(-E_{t}\right)=\alpha_{1}\left((-1-\gamma)\left(-E_{t-1}\right)\right)^{\delta}=\alpha_{1}(1+\gamma)^{\delta}\left(E_{t-1}\right)^{\delta} \\
\left\{A C I_{A P A R C H}=\frac{\eta\left(E_{t}\right)-\eta\left(-E_{t}\right)}{\eta\left(E_{t}\right)+\eta\left(-E_{t}\right)}=\frac{\alpha_{1}(1-\gamma)^{\delta}-\alpha_{1}(1+\gamma)^{\delta}}{\alpha_{1}(1-\gamma)^{\delta}+\alpha_{1}(1+\gamma)^{\delta}}\right. \\
=\frac{(1-\gamma)^{\delta}-(1+\gamma)^{\delta}}{(1-\gamma)^{\delta}+(1+\gamma)^{\delta}}
\end{gathered}
$$

For the $\operatorname{EGARCH}(1,1)$ model the ACI can be derived as 


$$
\begin{aligned}
& \eta\left(E_{t}\right)=\left(\alpha_{1}+\phi_{1}\right) \frac{E_{t-1}}{\sigma}=\frac{\alpha_{1}+\phi_{1}}{\sigma} E_{t-1} \\
& \left\{\begin{array}{l}
\eta\left(-E_{t}\right)=\left(-\alpha_{1}+\phi_{1}\right) \frac{-E_{t-1}}{\sigma}=\frac{\alpha_{1}-\phi_{1}}{\sigma} E_{t-1} \\
A C I_{E G A R C H}=\frac{\eta\left(E_{t}\right)-\eta\left(-E_{t}\right)}{\eta\left(E_{t}\right)+\eta\left(-E_{t}\right)} \\
=\frac{\left(\alpha_{1}+\phi_{1}-\left(\alpha_{1}-\phi_{1}\right)\right) E_{t-1}}{\left(\alpha_{1}+\phi_{1}+\left(\alpha_{1}-\phi_{1}\right)\right) E_{t-1}}=\frac{2 \phi_{1}}{2 \alpha_{1}}=\frac{\phi_{1}}{\alpha_{1}}
\end{array}\right.
\end{aligned}
$$

Note that in all GARCH type models, the $A C I$ s are only governed by the parameter of variance equations. With ACIs, the asymmetry of the NIC can be measured quantitatively.

\section{Case study}

Firstly, the asymmetry effect in the volatility of wind power time series is witnessed by modeling asymmetric GARCH type models. News Impact Curve is applied to analyze the asymmetry characteristics in wind power series. Furthermore, the application of new notions, BSC and $A C I$, are illustrated. Finally, the forecasting precisions of all the asymmetric GARCH-type models are compared.

\subsection{Data description}

Considering that Yancheng, a city with abundant coastal wind resources in east China, is able to represent the pattern of wind power in East China, Data of wind farm groups in Yancheng, is used to collate the results generated by the asymmetric GARCH wind power forecasting models.

The sample originates from the wind power data between April 1 and April 7. The wind power data is captured every $5 \mathrm{~min}$, thus, there are 288 data points each day, and the overall data sample includes 2016 points of data. The statistics of the data is summarized in Table 1.

The 5-min forecasting of wind power on April 8 by GARCH type models is provided. Forecasting precisions of these models are verified by a comparison with the actual data, in terms of several statistical indices.

Table 1 The statistics of data

\begin{tabular}{ll}
\hline Statistics & $Y_{t}$ \\
\hline Mean & $65.5 \mathrm{MW}$ \\
Median & $63.5 \mathrm{MW}$ \\
Std. Dev. & $42.83 \mathrm{MW}$ \\
Skewness & 0.429 \\
Kurtosis & 2.516 \\
Observations & 2016 \\
\hline
\end{tabular}

\subsection{Modeling}

The results of an ADF test and PP test are consistent with the fact that the original wind power series $Y_{t}$ is not stationary at a $5 \%$ significance level.

Differencing is used to get the first-differenced series $I_{\mathrm{t}}$. At the $1 \%$ significance level, the statistics of the ADF test is statistically significant. Moreover, the result of PP test is in accord with the result of ADF test. Therefore, the stationarity of $I_{t}$ is verified and the stationary precondition of modeling is satisfied. The following modeling focuses on $I_{t}$.

\subsection{Parameter estimate}

The ARMA structure, including $p$ autoregressive (AR) and $q$ moving average (MA) terms, is used to depicted the conditional mean of the GARCH type model. The conditional mean equation of the ARMA-GARCH-M model is given as

$$
\begin{aligned}
I_{t} & =\omega-E\left(g\left(h_{t}\right)\right)+\sum_{j=1}^{p} \phi_{i} I_{t-i}+\varepsilon_{t}+\sum_{i=1}^{q} \theta_{i} \varepsilon_{t-i}+g\left(h_{t}\right) \\
& =\tilde{\omega}+\sum_{j=1}^{p} \phi_{i} I_{t-i}+\varepsilon_{t}+\sum_{i=1}^{q} \theta_{i} \varepsilon_{t-i}+g\left(h_{t}\right)
\end{aligned}
$$

Based on reference [7], the orders of model are determined as ARMA $(4,5)$-GARCH $(1,1)-\mathrm{M}$ with $g\left(h_{t}\right)=\delta h_{t}(\mathrm{GARCH}-$ $\mathrm{M}^{1}$, for short). Furthermore, the parameter set $\Theta_{\text {GARCH }}=(\vartheta$, $\left.\delta, \Omega, \alpha_{1}, \beta_{1}\right)$ is obtained by CMLE, where $\vartheta$ stands for the ARMA parameter subset in a conditional mean equation. Similarly, with the same routine, the GARCH-M $\mathrm{M}^{2}$ following (5) and the GARCH-M $\mathrm{M}^{3}$ following (6) can be obtained, respectively. Moreover, inducing the asymmetric GARCH structure,the GJR-M ${ }^{1}$, GJR-M ${ }^{2}$, GJR-M ${ }^{3}$, EGARCH-M ${ }^{1}$, EGARCH-M ${ }^{2}$, EGARCH-M ${ }^{3}$, APARCH-M ${ }^{1}$, APARCH-M ${ }^{2}$, and APARCH-M ${ }^{3}$ can be obtained.

Parameters of 9 asymmetric GARCH-M models estimated by CMLE, are shown in Table 2.

In view of the heavy tail effect in the wind power volatility, these asymmetric GARCH-M models are generalized to heavy tail versions. Estimates of the 9 asymmetric GARCH-M models with t- distribution and the 9 asymmetric GARCH-M models with GED are summarized in Table 3. Similarly, the standard error is presented in parentheses.

According to Table 1 and Table 2, it can be found that:

1) The witness of asymmetric conditional volatility is verified by the asymmetric parameter in every GARCH type model. According to Table 1, the estimated value of $\phi$ in (9) is $0.098227,0.095975$ and 0.095157 respectively; the estimated value of $\gamma$ in (7) is -0.11382 , -0.11407 , and -0.11595 respectively; the estimated 
Table 2 Parameter estimate of standard asymmetric GARCH

\begin{tabular}{|c|c|c|c|c|c|c|c|c|c|c|c|c|}
\hline & $\delta$ & $C$ & $\operatorname{AR}(1)$ & $\operatorname{AR}(4)$ & $M A(1)$ & MA(5) & $\Omega$ & $a_{1}$ & $\beta_{1}$ & $\varphi$ & $\gamma$ & $\delta$ \\
\hline \multirow[t]{2}{*}{$\overline{G J R}-M^{1}$} & -0.01707 & 0.138262 & 0.758611 & 0.071532 & -0.53293 & -0.05708 & 0.061024 & 0.152734 & 0.90498 & - & -0.1138 & - \\
\hline & $(0.011206)$ & $(0.127687)$ & $(0.059887)$ & $(0.028938)$ & $(0.070243)$ & $(0.026714)$ & $(0.020114)$ & $(0.01688)$ & $(0.009446)$ & - & $(0.01872)$ & - \\
\hline \multirow[t]{2}{*}{$G J R-M^{2}$} & -0.12493 & 0.252579 & 0.757966 & 0.071646 & -0.53252 & -0.05708 & 0.063384 & 0.151793 & 0.905159 & - & -0.1141 & - \\
\hline & $(0.066946)$ & $(0.135594)$ & (0.059805) & $(0.028851)$ & (0.070177) & $(0.026629)$ & $(0.020165)$ & $(0.016692)$ & $(0.009336)$ & - & $(0.01857)$ & - \\
\hline \multirow[t]{2}{*}{ GJR $-M^{3}$} & -0.15777 & 0.184332 & 0.754775 & 0.071773 & -0.52984 & -0.05666 & 0.06315 & 0.151475 & 0.906252 & - & -0.1160 & - \\
\hline & $(0.078565)$ & $(0.127137)$ & $(0.060031)$ & $(0.028712)$ & $(0.070462)$ & $(0.026597)$ & $(0.01984)$ & $(0.016569)$ & (0.009157) & - & $(0.01840)$ & - \\
\hline \multirow[t]{2}{*}{ APARCH-M } & 0.03011 & 0.189513 & 0.741602 & 0.066549 & -0.50437 & -0.06032 & 0.029162 & 0.065429 & 0.939531 & - & -0.7841 & 1.0121 \\
\hline & $(0.011153)$ & $(0.120606)$ & $(0.056915)$ & $(0.027478)$ & $(0.067943)$ & $(0.027301)$ & (0.008198) & $(0.007594)$ & (0.007298) & - & $(0.11821)$ & $(0.1362)$ \\
\hline \multirow[t]{2}{*}{ APARCH-M ${ }^{2}$} & -0.15607 & 0.333815 & 0.735154 & 0.069739 & -0.49993 & -0.05877 & 0.030289 & 0.06851 & 0.93594 & - & -0.7157 & 1.1144 \\
\hline & $(0.064421)$ & $(0.132455)$ & $(0.057355)$ & $(0.027481)$ & $(0.068379)$ & $(0.027259)$ & $(0.009412)$ & $(0.008408)$ & $(0.00783)$ & - & $(0.1200)$ & $(0.1502)$ \\
\hline \multirow[t]{2}{*}{ APARCH-M ${ }^{3}$} & -0.1463 & 0.228257 & 0.726652 & 0.072908 & -0.49291 & -0.05751 & 0.029773 & 0.07136 & 0.933465 & - & -0.6613 & 1.2000 \\
\hline & $(0.071718)$ & $(0.121132)$ & $(0.058073)$ & $(0.027401)$ & $(0.068928)$ & $(0.02723)$ & (0.010149) & $(0.008975)$ & $(0.00815)$ & - & $(0.1159)$ & $(0.1634)$ \\
\hline \multirow[t]{2}{*}{ EGARCH-M ${ }^{1}$} & -0.03180 & 0.184432 & 0.740065 & 0.068059 & -0.50353 & -0.06014 & -0.0732 & 0.12471 & 0.988158 & 0.09823 & - & - \\
\hline & $(0.01137)$ & $(0.117749)$ & $(0.056588)$ & $(0.027494)$ & $(0.067426)$ & $(0.027228)$ & $(0.011587)$ & $(0.014321)$ & $(0.003097)$ & $(0.01060)$ & - & - \\
\hline \multirow[t]{2}{*}{ EGARCH-M ${ }^{2}$} & -0.1428 & 0.317678 & 0.732846 & 0.069608 & $-0.49802)$ & -0.05928 & -0.08167 & 0.132828 & 0.98937 & 0.09598 & - & - \\
\hline & $(0.05914)$ & $(0.121551)$ & $(0.057383)$ & $(0.02746)$ & $(0.068276$ & (0.027198) & $(0.011812)$ & $(0.015118)$ & $(0.00285)$ & $(0.01079)$ & - & - \\
\hline \multirow[t]{2}{*}{ EGARCH-M ${ }^{3}$} & -0.1095 & 0.219511 & 0.724388 & 0.071589 & -0.49089 & -0.05744 & -0.08839 & 0.138758 & 0.990784 & 0.09516 & - & - \\
\hline & $(0.05682)$ & $(0.112735)$ & $(0.058415)$ & $(0.027348)$ & $(0.069065)$ & $(0.027307)$ & $(0.012083)$ & $(0.01567)$ & $(0.002564)$ & $(0.01082)$ & - & - \\
\hline
\end{tabular}

value of $\gamma$ in (8) is $-0.78405,-0.71568$, and -0.66128 , respectively. All asymmetric parameters are significant, and the existence of asymmetric volatility is witnessed. Moreover, owing to the definition of $\phi$ and $\gamma$, the physical meaning of asymmetric effect is consistent. That is to say, if the innovation is positive, the future volatility will be stronger than the case where the innovation is negative in the same magnitude. Note that the asymmetric effect of wind power time series is similar to the effect of load time series.

2) The estimated parameters, $\phi$ and $\gamma$, of the fat-tail asymmetric GARCH-M model in Table 2 are in accordance with the corresponding parameters of the GARCH-M model in Table 1. Despite significant difference among these models, The existence of asymmetric effect of the wind power time series is affirmed.

3) The values of GED shape parameters of all GED versions of asymmetric GARCH models between 1.56 and 1.57. In this case, GED has heavier tails than normal distribution. Moreover, the $\mathrm{t}$ distribution freedom parameters in the $t$ distribution version of asymmetric GARCH models are significant. Therefore, the heavy tail effect in wind power series is doubly confirmed.

\subsection{The application of NIC and BSC}

With the discussion in Section 3, the enhanced NIC is proposed to analyze the impact of positive and negative idiosyncratic shocks on the volatility of the wind power time series.
The choice of different volatility compensation terms has little influence on the shape of the NIC in a practical wind power time series. To simplify the discussion, only the type I GARCH-M structure, defined by (4), is employed in the mean equation, and NICs and BSCs of the asymmetric GARCH-M ${ }^{1}$ models are presented.

\section{1) GJR-M ${ }^{1}$}

To simplify the comparison, all the curves have been moved to the original point. According to Table 1, the equation of NICs of GJR-M ${ }^{1}$ models with normal distributions is shown as

$$
\left\{\begin{array}{l}
h_{t}=\alpha_{1} \varepsilon_{t-1}^{2}=0.1527 \varepsilon_{t-1}^{2} \quad \varepsilon_{t-1} \geq 0 \\
h_{t}=\left(\alpha_{1}+\gamma\right) \varepsilon_{t-1}^{2}=0.0389 \varepsilon_{t-1}^{2} \quad \varepsilon_{t-1}<0
\end{array}\right.
$$

According to the definition of BSC, the equation of GJR-M ${ }^{1}$ BSC that has been moved to the original point is shown as

$$
H_{t_{-} G J R}=h_{t}=\alpha_{1} \varepsilon_{t-1}^{2}=0.1527 \varepsilon_{t-1}^{2}
$$

Similarly, according to Table 1, the equations of NICs of GJR-M ${ }^{1}$ models with $\mathrm{t}$-distributions and GEDs are shown as

$$
\begin{cases}h_{t}=\alpha_{1} \varepsilon_{t-1}^{2}=0.1539 \varepsilon_{t-1}^{2} & \varepsilon_{t-1} \geq 0 \\ h_{t}=\left(\alpha_{1}+\gamma\right) \varepsilon_{t-1}^{2}=0.0429 \varepsilon_{t-1}^{2} & \varepsilon_{t-1}<0\end{cases}
$$




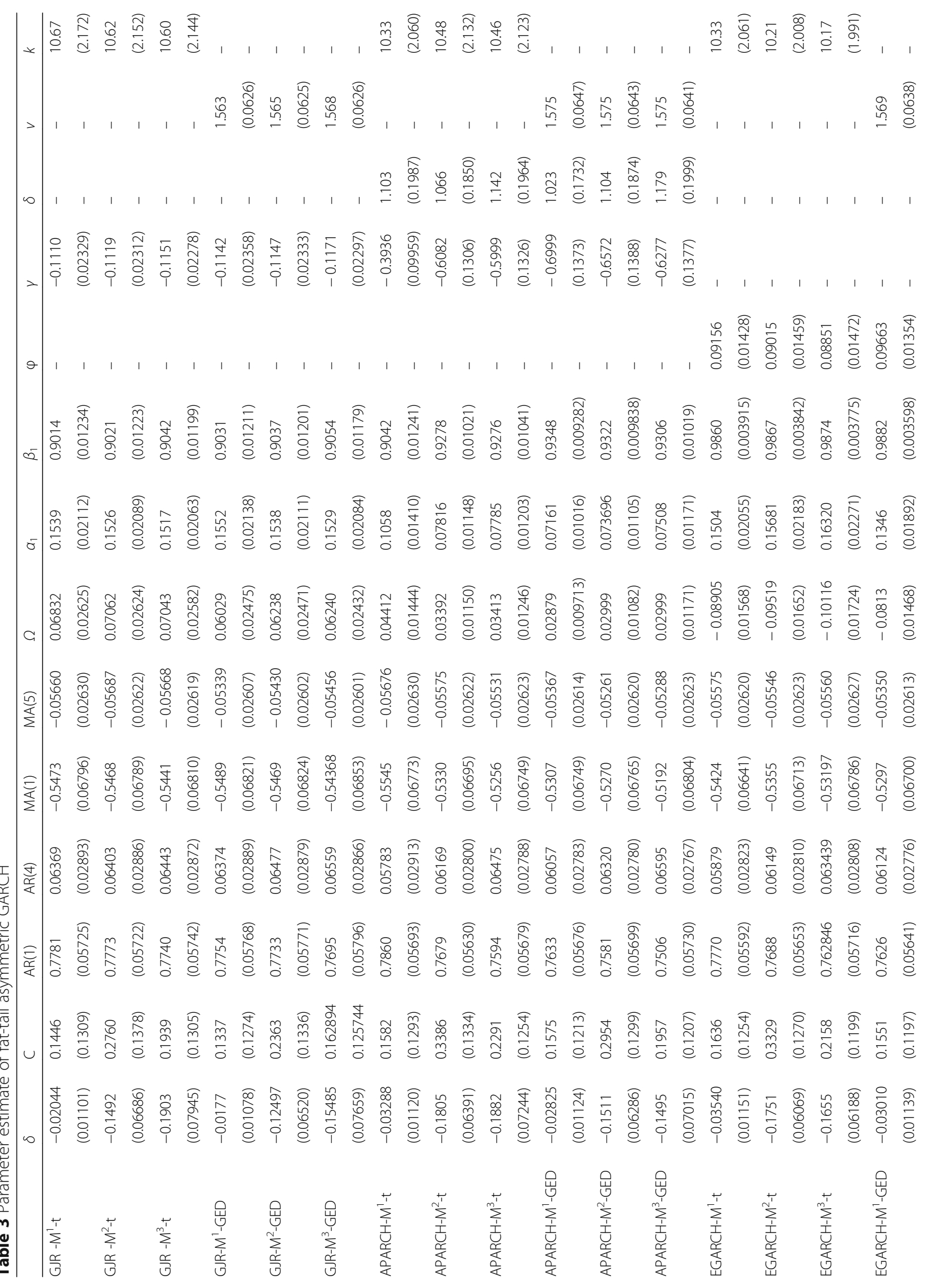




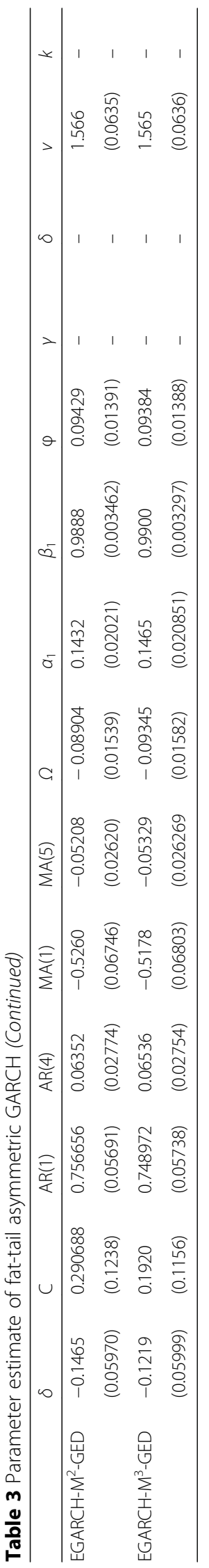




$$
\begin{cases}h_{t}=\alpha_{1} \varepsilon_{t-1}^{2}=0.1552 \varepsilon_{t-1}^{2} & \varepsilon_{t-1} \geq 0 \\ h_{t}=\left(\alpha_{1}+\gamma\right) \varepsilon_{t-1}^{2}=0.0410 \varepsilon_{t-1}^{2} & \varepsilon_{t-1}<0\end{cases}
$$

Furthermore, the NICs of GJR-M ${ }^{1}$ models with different distributions and the BSC of GJR-M ${ }^{1}$ are represented as Fig. 1, respectively.

As shown in Fig. $1, H_{t}$ is plotted against $\varepsilon_{t-1}$. The blue line, red line and green line stand for the NIC of a standard GJR-M ${ }^{1}$ model, GJR-M ${ }^{1}$ with $\mathrm{t}$-distributions and GJR-M ${ }^{1}$ with generalized error distributions, respectively. Note that black dotted line denotes the vertical axis of the NIC group.

The Benchmark Symmetric Curve of GJR acts as a benchmark. As illustrated in Fig. 1, the left branch of GJRGARCH _NIC is lower than the right branch. It is indicated that positive shocks impose more influence on volatility than negative shocks with the same magnitude. It can be found that the asymmetry of the NIC can be verified distinctly, with the help of the symmetric structure of the BSC.

\section{2) APARCH-M ${ }^{1}$}

Furthermore, the equation of NICs of APARCH-M ${ }^{1}$ models with normal distributions is shown as

$$
\left\{\begin{array}{cc}
h_{t}^{\delta} / 2=\alpha_{1}(1-\gamma)^{\delta} \varepsilon_{t-1}^{\delta}=0.1175 \varepsilon_{t-1}^{\delta} & \varepsilon_{t-1} \geq 0 \\
\delta / 2 & \\
h_{t} / 2=\alpha_{1}(1+\gamma)^{\delta}\left(-\varepsilon_{t-1}\right)^{\delta}=0.0139\left(-\varepsilon_{t-1}\right)^{\delta} & \varepsilon_{t-1}<0
\end{array}\right.
$$

According to the definition of $\mathrm{BSC}$, the equation of APARCH-M ${ }^{1} \mathrm{BSC}$ that has been moved to the original point is shown as

$$
H_{A P A R C H}=h_{t}^{\delta} / 2=\alpha_{1}=0.0654\left|\varepsilon_{t-1}\right|^{\delta}
$$

Similarly, According to Table 1, the equations of NICs of APARCH-M ${ }^{1}$ models with $t$-distributions and GED are shown as

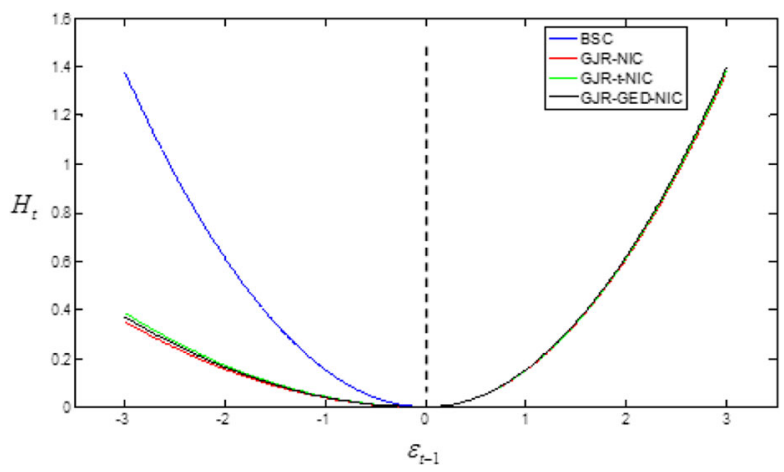

Fig. 1 The NICs and BSC of GJR-M1 models

$$
\begin{aligned}
& \left\{\begin{array}{l}
h_{t} / 2 \\
\delta / 2
\end{array} / \alpha_{1}(1-\gamma)^{\delta} \varepsilon_{t-1}^{\delta}=0.1526 \varepsilon_{t-1}^{\delta} \quad \varepsilon_{t-1} \geq 0\right. \\
& h_{t}^{\delta} / 2=\alpha_{1}(1+\gamma)^{\delta}\left(-\varepsilon_{t-1}\right)^{\delta}=0.0609\left(-\varepsilon_{t-1}\right)^{\delta} \quad \varepsilon_{t-1}<0
\end{aligned}
$$

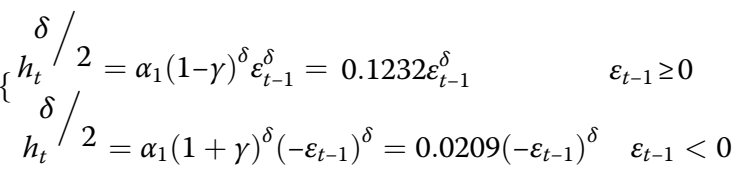

Furthermore, the NICs of APARCH-M ${ }^{1}$ models with different distributions and the BSC of APARCH-M $\mathrm{M}^{1}$ are represented as Fig. 2, respectively.

\section{3) EGARCH}

The equation of NICs of EGARCH-M ${ }^{1}$ models with normal distributions is shown as

$$
\begin{cases}\ln h_{t}=\frac{\alpha_{1}+\phi_{1}}{\sigma} \varepsilon_{t-1}=0.0627 \varepsilon_{t-1} & \varepsilon_{t-1} \geq 0 \\ \ln h_{t}=\frac{-\alpha_{1}+\phi_{1}}{\sigma} \varepsilon_{t-1}=-0.0075 \varepsilon_{t-1} & \varepsilon_{t-1}<0\end{cases}
$$

According to the definition of $\mathrm{BSC}$, the equation of EGARCH-M ${ }^{1}$ BSC that has been moved to the original point is shown as

$$
H_{E G A R C H}=\ln h_{t}=\frac{\alpha_{1}}{\sigma}\left|\varepsilon_{t-1}\right|=0.0351\left|\varepsilon_{t-1}\right|
$$

Similarly, according to Table 1, the equations of NICs of EGARCH $-\mathrm{M}^{1}$ models with $\mathrm{t}$-distributions and GEDs are shown as.

$$
\begin{cases}\ln h_{t}=\frac{\phi_{1}+\alpha_{1}}{\sigma} \varepsilon_{t-1}=0.0681 \varepsilon_{t-1} & \varepsilon_{t-1} \geq 0 \\ \ln h_{t}=\frac{\phi_{1}-\alpha_{1}}{\sigma} \varepsilon_{t-1}=-0.0165 \varepsilon_{t-1} & \varepsilon_{t-1}<0\end{cases}
$$

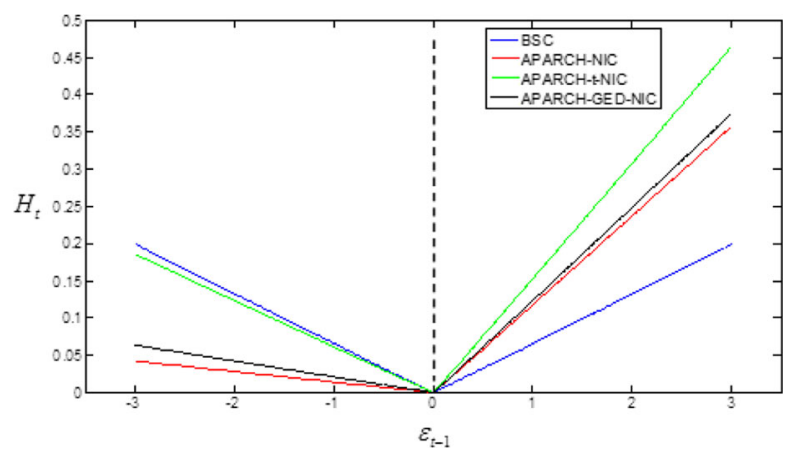

Fig. 2 The NICS and BSC of APARCH-M1 models 


$$
\left\{\begin{array}{lr}
\ln h_{t}=\frac{\phi_{1}+\alpha_{1}}{\sigma} \varepsilon_{t-1}=0.0650 \varepsilon_{t-1} & \varepsilon_{t-1} \geq 0 \\
\ln h_{t}=\frac{\phi_{1}-\alpha_{1}}{\sigma} \varepsilon_{t-1}=-0.0107 \varepsilon_{t-1} & \varepsilon_{t-1}<0
\end{array}\right.
$$

Furthermore, the NICs of EGARCH $-\mathrm{M}^{1}$ models with different distributions and the BSC of EGARCH $-\mathrm{M}^{1}$ are demonstrated in Fig. 3, respectively.

Note that the curves of EGARCH are nondifferentiable at the lowest point and the curves are evidently skewed towards left.

The results by NICs of GJR-M ${ }^{1}$ are confirmed with the NICs of the APARCH $-\mathrm{M}^{1}$ and EGARCH $-\mathrm{M}^{1}$ models. It is identified that asymmetric effect basically remains stable in spite of different options of specification. In particular, all the curves reflect an obvious shift to the left. Through the application of the NIC and BSC, how the asymmetric effects between different shocks work is highlighted clearly.

\subsection{Application of $\mathrm{ACl}$}

With the help of (20), (21) and (22), the calculated $A C I$ value of each model is summarized in Table 4 .

As can be seen from Table 4 the asymmetry of the NIC is measured quantitatively. It is apparent that the three conditional distributions specification of the same model induce remarkably different $A C I$ values. The influence of conditional distribution on the $A C I$ cannot be ignored.

Take the GJR-M model as an example: the $A C I$ of GJR-M ${ }^{1}$ with different conditional distribution is 0.594, 0.564 and 0.582 , respectively. The $A C I$ of GJR- $\mathrm{M}^{1}$-t has the least value-that is to say, the asymmetry of NIC_ GJR- $\mathrm{M}^{1}-\mathrm{t}$ is the weakest.

Furthermore, it appears that the GARCH type models with $t$-distribution often relate to a comparatively low $A C I$ value in the result. All $A C I$ s of the above GARCH type models with $t$-distribution have the lowest value (that is, $0.564,0.429$ and 0.609 respectively), while the NICs of models with normal

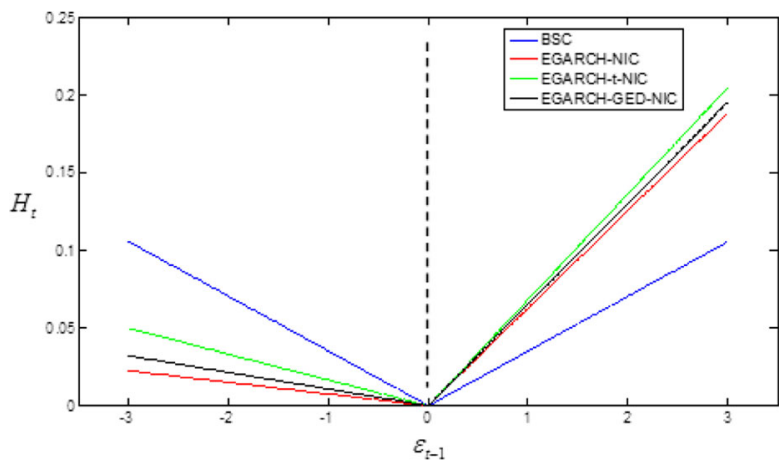

Fig. 3 The NICs and BSC of EGARCH-M1 models
Table $4 \mathrm{ACl}$ value of Asymmetric GARCH-M1 Models

\begin{tabular}{|c|c|c|c|c|c|c|c|c|c|}
\hline \multicolumn{4}{|c|}{ GJR-M ${ }^{1}$} & \multicolumn{3}{|c|}{ APARCH-M ${ }^{1}$} & \multicolumn{3}{|c|}{ EGARCH-M ${ }^{1}$} \\
\hline & Normal & $t$ & G & Normal & $t$ & G & Normal & $t$ & G \\
\hline$\overline{A C l}$ & 0.594 & 0.564 & 0.582 & 0.789 & 0.429 & 0.710 & 0.788 & 0.609 & 0.718 \\
\hline
\end{tabular}

Bold entries are all ACls with t-distribution that have the lowest value

distribution are on the contrary. The significant asymmetry of the above models could also be recognized by the NICs.

\subsection{Forecasting performance}

The wind power forecasting formula is given by

$$
\hat{Y}_{t}=Y_{t-1}+\hat{I}
$$

where $\hat{I}$ are modeled by the asymmetric GARCH type models, respectively. The GARCH model proposed by [16], classical ARMA model are used as the benchmark model. Based on these forecasting models mentioned, $5 \mathrm{~min}$ wind power forecasting results for the coming $24 \mathrm{~h}$ are given. Several classical criteria, $E_{\mathrm{RMSE}}$ (Root Mean Squared Error), $E_{\mathrm{MAE}}\left(\right.$ Mean Absolute Error) and $E_{\mathrm{MAPE}}$ (Mean Absolute Percentage Error), are listed in Table 5.

After analyzing Table 5, it turns out that the APARCH-M ${ }^{1}$ model renders the best performance. Fig. 4 illustrates the out-of-sample forecasts by APARCH-M ${ }^{1}$ model, and the upper and lower limits of the prediction interval is also presented.

Furthermore, the forecasting precisions of asymmetric GARCH-M models and their generalized version are satisfied and better than the traditional GARCH-M model.

\begin{tabular}{|c|c|c|c|}
\hline Name & $E_{\text {RMSE }}(M W)$ & $E_{\text {MAE }}(M W)$ & $E_{\text {MAPE }}(\%)$ \\
\hline$\overline{\mathrm{TP}}$ & 16.4495 & 12.0368 & 17.468 \\
\hline ARMA & 5.1308 & 3.4087 & 4.693 \\
\hline GARCH-M ${ }^{1}$ & 5.1023 & 3.3850 & 4.678 \\
\hline GARCH-M ${ }^{2}$ & 5.1183 & 3.400 & 4.684 \\
\hline GARCH-M ${ }^{3}$ & 5.1242 & 3.4066 & 4.686 \\
\hline GJR-M ${ }^{1}$ & 5.0891 & 3.3777 & 4.657 \\
\hline GJR-M ${ }^{2}$ & 5.1034 & 3.3859 & 4.656 \\
\hline GJR-M $M^{3}$ & 5.1087 & 3.3903 & 4.655 \\
\hline EGARCH-M ${ }^{1}$ & 5.0821 & 3.3549 & 4.632 \\
\hline EGARCH-M ${ }^{2}$ & 5.1014 & 3.3753 & 4.641 \\
\hline EGARCH-M ${ }^{3}$ & 5.1037 & 3.3823 & 4.647 \\
\hline APARCH-M ${ }^{1}$ & 5.0791 & 3.3521 & 4.630 \\
\hline APARCH-M ${ }^{2}$ & 5.1006 & 3.3741 & 4.640 \\
\hline APARCH-M ${ }^{3}$ & 5.1041 & 3.3821 & 4.646 \\
\hline
\end{tabular}

Table 5 Forecasting precision of proposed models

Bold entries are value in APARCH-M1 


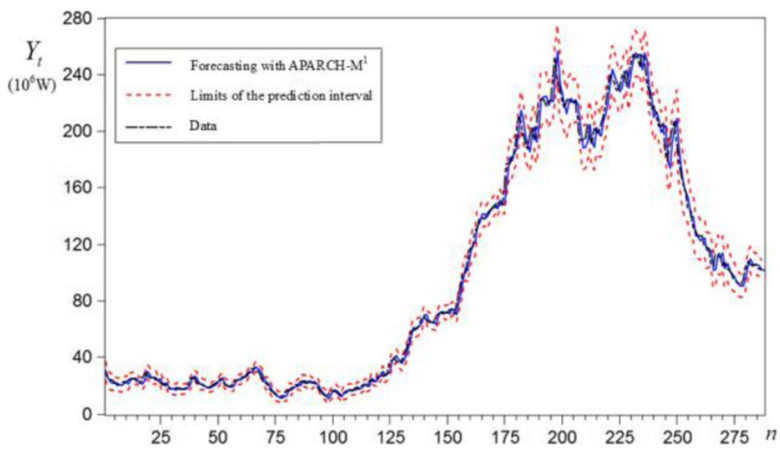

Fig. 4 the forecast by APARCH-M ${ }^{1}$ model

\section{Conclusions}

In this paper, an investigating framework for considering the impact of news on volatility in wind power is provided.

First, the asymmetric effect on wind power volatility is reconfirmed. With the asymmetric GARCH model, it is evident that the impacts of negative shocks on volatility are weaker than positive shocks, ceteris paribus.

Second, the Benchmark Symmetric Curve is proposed as the new benchmark, which gives an analytical framework to compare the NIC of asymmetric GARCH models.

Third, the Asymmetric Curve Index (ACI) provides a corresponding quantitative measure to the asymmetry of the NIC. The case study validated the efficiency of an ACI.

In summary, the refined NIC analysis is advocated to describe heteroskedasticity. Thanks to the GARCH models, the theoretical analysis on asymmetric volatility in wind power time series can contribute to a further perception of some intrinsic features of wind power and considerably improve the forecasting precision.

\section{Abbreviations}

ACl: Asymmetric Curve Index; BSC: Benchmark Symmetric Curve; NIC: News Impact Curve

\section{Acknowledgements}

Not applicable.

\section{Authors' contributions}

HC carried out the Asymmetric GARCH Type Models studies, participated in the Benchmark Symmetric Curve (BSC) and Asymmetric Curve Index (ACl) design and drafted the manuscript. JZ helped to draft the manuscript part III. $Y T$ participated in the design of the study and performed the statistical analysis. FT helped design of the statistical analysis. All authors read and approved the final manuscript.

\section{Funding}

This work is funded by National Natural Science Foundation of China (51577025).

\section{Availability of data and materials}

Data sharing not applicable to this article as no datasets were generated or analyzed during the current study.

\section{Competing interests}

The authors declare that they have no competing interests.

\section{Author details}

${ }^{1}$ State Grid Jiangsu Electric Power Co., Ltd Maintenance Branch Company, Nanjing 211102, Jiangsu, China. ${ }^{2}$ Southeast University, Nanjing 210096,

Jiangsu, China.

Received: 2 May 2019 Accepted: 26 November 2019

Published online: 30 December 2019

\section{References}

1. Ding, T., Wu, Z., Lv, J., et al. (2016). Robust co-optimization to energy and ancillary service joint dispatch considering wind power uncertainties in realtime electricity markets. IEEE Transactions on Sustainable Energy, 7(4), 15471557

2. Li, J., Wang, S., Ye, L., et al. (2018). A coordinated dispatch method withpumped-storage and battery-storage forcompensating the variation of wind power protection and control of modern power systems. Protection and control of modern power Systems, 3(2), 1-14.

3. Global wind report annual market update 2017. http://files.gwec.net/ register/success/?file=/files/GWR2017.pdf. Accessed 2 Apr 2018.

4. Ye, R., Suganthan, P. N., \& Srikanth, N. (2015). Ensemble methods for wind and solar power forecasting-a state-of-the-art review. Renewable and Sustainable Energy Reviews, 50, 82-91.

5. KLange, M., \& Focken, U. (2009). Physical approach to short term wind power prediction. New York: Springer-Verlag.

6. Alexiadis, M. C., Dokopoulos, P. S., \& Sahsamanoglou, H. S. (1999). Wind speed and power forecasting based on spatial models. IEEE Transactions on Energy Conversion, 14(3), 836-842.

7. Taylor, J. W., McSharry, P. E., \& Buizza, R. (2009). Wind power density forecasting using wind ensemble predictions and time series models. IEEE Transactions on Energy Conversion, 24, 775-782.

8. Chen, H., Wan, Q., Li, F., et al. (2013). GARCH in mean type models for wind power forecasting. Vancouver: Proceedings of the IEEE PES General Meeting 24-29 July 2013

9. Louka, P., Galanis, G., Siebert, N., et al. (2008). Improvements in wind speed forecasts for wind power prediction purposes using Kalman filtering. Journal of Wind Engineering and Industrial Aerodynamics, 96(12), 2348-2362.

10. Zheng, D., Eseye, A. T., Zhang, J., et al. (2017). Short-term wind power forecasting using a double-stage hierarchical ANFIS approach for energy management in microgrids. Protection and Control of Modern Power Systems, 2, $1-10$.

11. Catalao, J. P. S., Pousinho, H. M. I., \& Mendes, V. M. F. (2011). Short-term wind power forecasting in Portugal by neural networks and wavelet transform. Renewable Energy, 36, 1245-1251.

12. Bin, L. I., Shurong, P., Junzh, P., et al. (2018). Wind power probability density forecasting based on deep learning quantile regression model. Electric power automation Equipment, 38(9), 15-20.

13. Jun, L., \& Dachao, L. (2016). Wind power time series prediction using optimized kernel extreme learning machine method. Acta Physica Sinica, 65(13), 39-48.

14. Hongyu, Z., Qian, Z., Wang, Z., et al. (2014). Very short-term wind turbine output forecasting with compositional model. Acta energiae solaris sinica 35(3), 457-461.

15. Engle, R., \& Ng. (1993). Measuring and testing the impact of news on volatility. The Journal of Finance, 48(5), 1749-1778.

16. Bollerslev. (1986). Generalized autoregressive conditional Heteroskedasticity. Journal of Econometrics, 31, 307-327.

17. Glosten, L., Jagannathan, R., \& Runkle, D. (1993). On the relation between the expected value and volatility of the nominal excess return on stocks. The Journal of Finance, 48(5), 1779-1801.

18. Ding, Z., Granger, C. W. J., \& Engle, R. (1993). A long memory property of stock market returns and a new model. Journal of Empirical Finance, 1, 83-106.

19. Nelson, D. B. (1991). Conditional heteroskedasticity in asset returns: A new approach. Econometrica, 59(2), 347-370.

20. Fan, J., \& Yao, Q. (2003). Nonlinear time series: Nonparametric and parametric methods (pp. 125-192). New York: Springer-Verlag.

21. Tsay, R. S. (2012). An introduction to analysis of financial data with $R$ (pp. 176-273). Hoboken: Wiley. 